\title{
Nathalie Quintane et la part sombre de l'histoire de France
}

\author{
Alain Farah \\ Université McGill
}

When we started this program, I don't know how many hours ago, Rich, the one thing that was evident is that the NY Giants were playing New England Patriots and New England Patriots were playing against History. And when I thought about that, I said you know it's gonna be tough. History isn't a good opponent. If you can't lead it, if you can't fight it, it doesn't fight you back. The only thing you are doing is giving the opponent some good material.

Un commentateur américain, à la suite de la défaite crève-cœur des Patriots de la Nouvelle-Angleterre au Super Bowl XLII, défaite mettant un terme à une séquence de seize victoires consécutives, une première dans la NFL. 
De livre en livre, Nathalie Quintane réactualise une position de résistance et d'invention dans le champ littéraire français. Si on a l'habitude de considérer son travail comme une critique d'une poésie prisonnière de l'illusion de la densité et de la profondeur, il est plus rare d'aborder son œuvre du point de vue d'une réflexion sur l'histoire. Si l'indication (entendue presque comme slogan littéraliste) posée en quatrième de couverture du deuxième livre de l'auteur prétend que "Chaussure parle vraiment de chaussure », cette détermination à revendiquer le premier degré n'empêche pas le passé de montrer son visage, sorte de rappel que le monde n'est jamais loin, que la limite du projet des pratiques littérales se situe dans leur rapport au politique. On peut certes résister à l'intimidation de pratiques trop spéculatives en rappelant les vertus critiques de la littéralité, mais il est difficile de postuler sérieusement que le langage est une fin en soi. Chaussure fait l'expérience de ce paradoxe : si les deux premières parties du livre examinent l'objet d'étude en évoquant des aspects matériels, techniques et anecdotiques qui concernent la chaussure, la troisième est marquée par l'entrée en scène de l'histoire, cette dernière étant soudain mise « au pied » du projet de Quintane :

Les sandales des anciens Égyptiens étaient fabriquées en papyrus; ils pouvaient donc marcher confortablement, sans se blesser quand la chaussure était neuve, et même utiliser celle-ci comme écritoire, y inscrire leur nom: «ces chaussures appartiennent à : », leur adresse, ou s'en servir de pense-bête : «ne pas oublier de rendre les dix drachmes au Grec », ou afficher leurs goûts, artistiques : «Le sphinx untel est une horreur », ou politiques : «J'ai cessé de soutenir untel », ou marcher sur un adversaire particulièrement haï, en plaçant son nom sous la semelle, ou adorer un dieu, en inscrivant son nom le plus haut possible sur les bribes. (1997, p. 73) 
Pour mener à bien son enquête, Quintane fait appel à l'époque pharaonique, à l'Antiquité grecque -Phiddipidès, Edipe ou Socrate : «Si Socrate marchait sans chaussures, habituellement, la corne qu'a dû se faire Socrate » (1997, p. 75) — et romaine — «D'une certaine manière, Caligula s'appelait chaussure (enfant il portait souvent une petite caliga — sandale) » $(1997$, p. 74) — de même qu'au Christ et à la Vierge. Dans «En Transcaucasie, les gens aussi ont des chaussures... », texte assommant où elle énumère les coutumes nationales de dizaines de pays relativement à la chaussure, Quintane fait entrer bien des nations dans son projet. Mais lorsque l'histoire contemporaine se montre, on perçoit le début d'une articulation entre le projet poétique et le projet politique, encore en germe dans Chaussure. En effet, l'écrivaine ne présente pas seulement des figures mythiques du passé, mais aussi des personnages ayant marqué les dernières décennies, comme à la fin de cette partie portant sur les liens entre chaussure et histoire :

Kroutchev était alors assis à une table. Il avait pour ainsi dire l'allure d'un homme important, son air. Aussi était-il, en effet, un homme important. [...] Or, soudain, Kroutchev avait mis sa chaussure sur la table, et tapé à plusieurs reprises avec celle-ci (toujours sur la table), si bien qu'on ne vit plus qu'elle. Soudain, sa chaussure avait remplacé Kroutchev: une chaussure de ville, montante, presque un godillot, sans caractéristiques particulières, bref, le type même du soulier banal, la plus ordinaire des chaussures. Or, Kroutchev tapait du pied, cette chaussure à la main, ou plutôt, la chaussure de Kroutchev tapait du pied pour lui. $\mathrm{Si}$ bien que Kroutchev disparaissait, momentanément, derrière. Non qu'il ait effectivement disparu, la chaussure ayant pris, par son élévation à table, des proportions hors normes, mais, aux yeux du spectateur, son air, lui, avait bel et bien disparu, brouillé par le surgissement impromptu d'un objet certes banal, mais déplacé. (1997, p. 84)

L'épisode relaté par Quintane est mémorable : le 12 octobre 1960, en pleine guerre froide, à l'Assemblée générale des Nations Unies, 
Nikita Kroutchev manifeste son mécontentement quant aux liens entre l'URSS et l'Europe de l'Est en prenant sa chaussure dans ses mains et en la cognant sur la table. Voilà un événement qui convient, dans sa curiosité, à l'esthétique de Quintane. Ce qu'elle en dit dans Chaussure va loin : Kroutchev est important comme homme politique seulement jusqu'à ce qu'il prenne sa chaussure pour manifester sa colère. À partir de ce moment, «la plus ordinaire des chaussures » éclipse le puissant. L'intérêt réside dans la capacité d'un objet à déloger l'indélogeable. L'apparition de la chaussure de Kroutchev aux Nations Unies est aussi imprévue que celle du premier secrétaire du Parti communiste dans un projet littéraire qui insiste pourtant pour dire qu'il ne parle que de chaussure. L'intérêt pour cet exemple est double. D'abord, il incarne une idée que Quintane ne cessera de développer dans ses livres suivants : il en faut peu pour mettre à mal les ordonnancements de la mémoire, pour montrer la part de cocasserie et de ridicule des figures du pouvoir. Dans le cas Kroutchev, une chaussure suffit à faire disparaître l'homme d'État. En même temps, à l'échelle des préoccupations littéraires de Quintane, la seule raison qui invite Kroutchev dans son livre est bel et bien cette chaussure. Chaussure avance ainsi au pas d'une glose littérale, certes, mais qui ne se gêne pas pour marcher au passage sur l'histoire et ses figures, à condition qu'elles se soumettent au dénominateur commun qu'est la chaussure. L'Histoire, avec sa majuscule, sa matière lourdement connotée, ses enflures, ses exaltations, est ramenée au ras du sol par un travail qui matérialise le conseil qu'un des personnages de Quintane donne à Antonia Bellivetti, protagoniste du roman (pour la jeunesse!) qui porte son nom : "Il ne faut pas être qu'un récipient des fables qui t'absorbent: histoire de ton père, histoires scolaires, hystérie de l'Histoire ». (Quintane, 2004, p. 109) Quintane œuvre ainsi à déplacer la matière historique, à la faire tourner sur elle-même, à la re- 
passer, c'est-à-dire aussi bien à mettre à plat sa grandiloquence qu'à revisiter l'histoire pour la faire voir autrement. Ainsi, cette narratrice faussement ingénue et si intensément engagée dans l'examen de la chaussure est aussi douteuse, puisqu'elle nivelle tout pour le bien de sa cause, au point qu'on observe sans l'ombre d'un scrupule les chaussures d'Imelda Marcos, l'épouse du dictateur philippin. En indiquant sans aucune autre explication qu' «Imelda Marcos a une collection de chausse-pieds en corne, en acajou, en lapis-lazuli, en ivoire et en ébène » (1997, p. 86), Quintane oblige le lecteur à faire face aux limites de la littéralité, limites à la fois politiques et éthiques. Car peut-on simplement parler des choses en tant que telles, sans réfléchir aux implications plus générales qui les concernent? Si le désir de densité est une illusion, la volonté de rester à la surface des choses est peut-être un fantasme. L'apparition d'Imelda Marcos dans Chaussure montre que les mots d'ordre, les stratégies de positionnement dans un champ atteignent vite leurs limites. Il n'y a pas de démarche d'écriture sérieuse qui ne soit contrainte de penser aussi à son déploiement dans le monde. Ces exemples révèlent que Quintane, réfractaire aux assignations de tout genre, s'intéresse à la littéralité non pas tant comme programme mais comme procédé et comme posture. Cette tangente se confirme avec Jeanne Darc : l'écrivaine assume qu'elle travaille désormais à faire entendre des voix.

\section{Plus les batailles s'accumulent, plus ma virginité s'étend}

Dans Saint-Tropez - Une Américaine, Quintane écrit : «C'est un bon sujet parce que ça n'en est pas un ». (2001, p. 34) L'inverse est aussi vrai : c'est un sujet trop mauvais (entendre nuisible) pour que je ne travaille pas dessus. Imagine-t-on, en effet, question plus pernicieuse que celle de Jeanne d'Arc, figure française par excellence, 
cambriolée depuis longtemps par l'extrême droite et le Front national de Jean-Marie Le Pen? Après Remarques et Chaussure, Jeanne Darc est une première occasion pour Quintane de travailler au « repassage » de l'histoire, à la reconsidération de ses icônes, de ses lieux, de ses événements, manière de lui faire subir une cure d'amaigrissement qui pointe le caractère friable, l'aspect fallacieux de son apparente incontestabilité. Quintane, en choisissant ce sujet connoté, chargé, ampoulé, élabore une enquête qui cherche à déboulonner la statue de la Pucelle. Contre la propagande, la précision : Quintane œuvre à retrouver l'équilibre entre la relativisation excessive et l'enflure hystérique de l'événement. À la fois reconstitution, mise en scène du mythe, récit et mise au point, Jeanne Darc donne la parole au personnage éponyme depuis un lieu précédant sa canonisation, la transformation de sa vie en mythe, comme pour rappeler qu'en cours de route, la petite Jeanne s'est « bonifiée ». En traitant d'un sujet à la fois convenu et délicat, Quintane sait qu'elle s'attaque, dans un contexte idéologique réfractaire à la contrition, à la part sombre de l'histoire de France, un «abject quelconque » qu'elle prend comme objet d'étude, tentant de voir ce qu'il recèle ou, au contraire, jusqu'à quel point il peut se montrer sans choquer. Pour ce faire, il faut aborder les objets historiques « délicats » exactement de la même façon qu'on traite une vulgaire chaussure. En somme, travailler différemment le politique, de biais, sans grandiloquence, posture emblématique de l'état d'esprit d'écrivains venus au monde « après 1968 ».

Sainte patronne de France, Jeanne d'Arc (1412-1431) représente à la fois la piété, la monarchie et l'unité nationale. Récupérée idéologiquement depuis des siècles (bien que ces tentatives soient souvent fondées sur des erreurs de perspectives historiques), elle est aussi depuis longtemps une inspiration pour les créateurs, de Vol- 
taire à Schiller en passant par Carl Theodor Dreyer, sans parler des adaptations cinématographiques plus récentes de sa vie, celle de Luc Besson notamment. La Jeanne Darc de Quintane est différente, et cela ne tient pas seulement à une question d'apostrophe. Le livre se divise en de courts épisodes qui reprennent et développent des éléments de la vie de la Pucelle. Travaillant à partir d'une matière première n'ayant rien d' « objectif » (matériaux « déjà mâchés » et aseptisés, c'est-à-dire, pour l'essentiel, des livres pris dans la bibliothèque de sa petite municipalité de Digne-les-Bains), Quintane, sans aucune intention historiographique, tente un périlleux exercice qui vise à dresser en contours la vie du personnage. Pour y parvenir, elle emploie plusieurs modalités et dispositifs formels qui colorent son examen : des vers, des anecdotes, des micro-récits, de la glose, des prières, et même un montage à partir de citations du Macbeth de Shakespeare ou encore une interview fictive de la Pucelle, qui n'échappe pas, sous la plume de Quintane, aux frasques de la société du spectacle.

Le livre commence par deux pages où flottent de nombreuses phrases, étrange chœur des voix entendues par Jeanne, exergue multiple expliquant à l'héroïne en quoi consiste sa mission : «Jeanne, tu dois aller à Vaucouleurs, rencontrer / Robert de Baudricourt, être conduite auprès de / Charles VII, le convaincre de ta mission, être / mise à la tête d'une petite troupe armée, obliger / les Anglais à lever le siège d'Orléans, et faire / sacrer le roi à Reims. » (1998, p. 7) L'écrivaine met en place une Pucelle tendue, toute constituée, pour reprendre une expression chère à Claude Duchet, d'«espaces conflictuels ${ }^{1} »$. En repérant ces binômes antithétiques,

1 Je rappelle cette citation de Claude Duchet : «Effectuer une lecture sociocritique revient, en quelque sorte, à ouvrir l'œuvre du dedans, à reconnaître ou à produire un espace conflictuel où le projet créateur se heurte à des résis- 
on voit à quel point Quintane veut faire de sa Jeanne un personnage divisé. Ce dualisme est repérable entre la pensée et l'action, les anecdotes et l'histoire, le baroque et l'austérité, la voix et l'écriture, l'ordre et le désordre. Bien sûr, si on doit n'en choisir qu'une seule, c'est l'opposition entre la bergère (exemple de l'anonymat, de l'individu sans singularité) et la soldate (le mythe qui sauve la France) qui est la plus féconde, puisqu'elle incarne aussi une dichotomie récurrente dans cet ouvrage, celle qui oppose l'idiotie des faits à la grandiloquence des récits historiques et idéologiques. Quintane, si elle commence le livre en présentant la Pucelle comme une bergère consacrée à sa tâche bien qu'impatiente «qu'il se passe quelque chose » $(1998$, p. 9), signale rapidement cette tension constitutive du personnage, pris entre «deux vies » (p. 16). Alternant les descriptions de la bergère et de la soldate, Quintane dresse le portrait d'une Jeanne qui ne cherche pas à résoudre cette ambivalence, comme pour montrer que la «mue » (p. 16) entre les deux états ne se fait pas proprement, demeure étrange, même pour la principale intéressée :

Pour quelle raison elle debout les bras ballants la contourne-t-on alors qu'assise à traire ou à tirer un fil d'un bouton, on s'arrête pour le seul recul de la voir faire; il n'y a qu'un bout de son propre passé surpris à l'œuvre chez un autre qui emporte à ce point? Pourquoi, en quoi, l'action de base (bouchonner, fourbir) ne suffisait pas? Pourquoi, en quoi, la contemplation de base (bruit de la pluie, poussière de pissenlit) ne suffisait pas non plus? Que faire pour que l'action soit encore plus agissante, et pour que la contemplation soit encore plus contemplante? (p. 23)

L'opposition bergère/soldate est ici augmentée de celle qui confronte la contemplation à l'action. Quintane met en place les élé-

tances, à l'épaisseur d'un déjà là, aux contraintes d'un déjà fait, aux codes et modèles socio-culturels, aux exigences de la demande sociale, aux dispositifs institutionnels. » $(1979$, p. 4) 
ments qui lui permettent de penser la question de la bonification, qui rend justement «l'action plus agissante», « la contemplation plus contemplante ». Elle insinue que ce pouvoir du mythologique de bonifier le passé peut occulter totalement la nature première des éléments qui font l'objet de cette bonification. Il s'agit de montrer qu'avant d'être soldate, Jeanne d'Arc était bergère, tout en donnant une fluidité entre ces pôles, preuve qu'ils ne sont pas aussi séparés :

Bien avant de faire le plan d'un trajet, de ma première campagne, d'une bataille, je préparais déjà, la veille, la nuit dans notre lit, le matin au réveil, la route du troupeau, la laine à filer, et je voyais les œufs s'ouvrir avant qu'ils tombent dans la poêle, et mon ongle tâchant de ne pas couper le jaune. Je n'ai jamais cousu un bouton sans m'être vue, avant, coudre le bouton. (p. 37)

De tout temps, donc, Jeanne a été prévoyante, vigilante : cette compétence en planification la mène «naturellement» de la tête d'un troupeau à la tête d'une armée. Cet extrait relève aussi la dichotomie entre la pensée et l'action. De la même façon, Quintane insiste ici aussi sur la proximité bergère/soldate :

Ou, à l'intérieur de ces deux vies, disons, d'intensités diverses, les événements ne peuvent qu'être traités de la même façon: un assaut, comme un rassemblement de troupeau; les soldats qu'on calme, comme un chien qu'on rudoie (Jeanne s'adapte, mais ne change pas). Sa personnalité de gardeuse ne s'est pas détachée et perdue en cours de route, mais projetée, elle s'est étendue, bonifiée. (p. 38)

Le télescopage est intéressant: du troupeau à l'armée, les compétences de la bergère n'ont fait que s'étendre, bonifiées par le mythe. Malgré cela, les allers et retours entre les deux Jeanne sont complexes, à un point tel qu'elle peut se scinder ou s'engendrer ellemême, la partie «postérieure » relatée dans l'extrait faisant office de supplément mythologique : 
Par la transformation qu'elle s'était imposée (cheval, cheveux) par l'influence conjuguée de Michel et Catherine, elle, Jeanne Darc, s'est extraite, fille, d'une ancienne et plus enfantine Jeannette. C'est à présent la Jeanne soldate qui veille et surveille la gardeuse de moutons. Voilà pourquoi Jeanne étant fille de Jeanne, elle ne peut nommer sa fille future Jeanne sans se déposséder de sa propre partie postérieure. (p. 51)

Cette opposition récurrente entre la bergère et la soldate vise à montrer le travail de bonification effectué par l'histoire. Ce n'est cependant pas l'élément qui engendre le plus de tension : plus que tout autre texte de Quintane, Jeanne Darc porte sur la guerre. La métaphore militaire y est prise en charge, non pas sur le mode parodique comme dans Le Colonel des Zouaves, mais de façon sérieuse, Quintane en faisant un réel objet de réflexion sur la littérature et la vie. Car sans guerre, Jeanne d'Arc n'est guère plus qu'une bergère : «Avant mon premier assaut, il y avait un monde entre le monde et moi : les cloches sonnaient, mon père criait, les moutons bêlaient. » (p. 25) Même si elle entend des voix, c'est son engagement qui la fait exister. Bien sûr, il est tentant de considérer cet emploi du lexique militaire comme une métaphore de la position de Quintane dans le champ, elle qui réfléchit, par l'entremise de son personnage, à la question de l'invention: «Elle songe à de nouvelles formes d'assaut. Malheureusement, le nombre de ruses militaires est restreint; on n'obtient, au mieux, qu'une nouvelle association de ruses anciennes, l'emboîtement d'une ruse dans l'autre, ou un enchaînement inattendu. » (p. 26) On n'invente pas tant qu'on agence à nouveau, comptant sur l'effet de surprise pour accentuer le choc, même si Jeanne admet l'attrait de la nouveauté : «Je ne désespère pas d'ajouter au répertoire des ruses celle qui portera mon nom / Car la préparation à la guerre me donne le goût de l'invention. » (p. 27) La guerre donne aussi l'occasion d'inventer un personnage mythique, puisque c'est par l'entremise de son rôle de soldate que Jeanne se 
transforme de bergère en sainte. La guerre a cette vertu « de rendre les choses concrètes par l'action » (p. 27). Voilà qui confirme pourquoi l'écriture de Quintane travaille le «mauvais», ce qui confronte: en rendant possible le choc, on permet au nouveau d'advenir. Cependant, la guerre est aussi une machine à fiction, à idéologies: "Plus les batailles s'accumulent, plus ma virginité s'étend» (p. 27), preuve que la vertu est soumise aux armes. Quintane ne s'en formalise pas trop: «Si tu n'aimes pas la guerre, change de guerre $»($ p. 27), dit-elle comme pour rappeler que rien n'oblige à la confrontation. La guerre a la capacité de modifier les événements, de mettre les gens à la fois sur le mode de l'attaque et de la défense, d'engendrer de l'inventivité. La guerre, métaphoriquement du moins, ouvre l'accès à l'imaginaire, permet de se frotter au monde. Voilà pourquoi les temps pacifiés sont propices à la complaisance et à la stagnation :

Le niveau de guerre dans la vie sans guerre est moindre, quoique persistant, et sous d'autres formes. La guerre à l'état vif dresse Jeanne dans un air homogène jamais démenti, où elle se sent ni de trop ni manquante. Être sur son cheval galopant vers une ville à ouvrir affirme et soi et le paysage - l'herbe est plus verte que le vert, les maisons, même la plus décatie des cabanes, sont maisons, on se figure leur intérieur, chaises et table dans l'unique pièce au sol battu, tout en passant au galop devant les traits verticaux du mur en lattes. Avant la guerre, et avant la définition qu'elle confère, la maison est opaque, celui qui y vit et à qui elle appartient en ferme l'accès, même au pillage de l'imagination. (p. 42)

Quintane ne fait pas pour autant l'apologie de la guerre, à la manière de Marinetti, souvent considéré comme le premier avantgardiste. Elle sait pertinemment qu'elle sert surtout la cause de la grandiloquence : «J'ai mis quelque temps à découvrir que la clarté de la guerre ne résidait pas dans l'assaut, mais dans le déroulement chronologique de la bataille remémorée. » (p. 60) Ainsi, elle met en 
lumière la part de fiction qui entoure chaque bataille, fiction devenue arme de combat au même titre que les autres, parce qu'elle donne un sens à l'événement, qu'elle le sépare de ce qu'il a été, qu'elle le rend plus gros, plus intimidant. Or, le travail politique de la littérature se situe exactement à l'inverse et consiste à montrer que peu de choses font l'histoire, que la contingence ne peut être cachée aux esprits critiques :

Passer des noms incertains de Tarc, Dare, Daire, de celle qu'on aurait pu ne pas nommer - elle aurait été la bergère, la fille de son père, une domrémique, une partisane -, à l'immunisé Darc Jeanne $[\ldots]$. Darc dupliquer, enfin au surnom, celui qu'on se rêve : Ange Bienfaiteur, Exterminateur, Blonde Protectrice, Gai et Patient Pourvoyeur, Aide au Salut, Lys Combattant, Fidèle Étendard, Indéfectible Adjuvant, Fleur Linéaire et Sage, l'Eil et l'Oreille, Autel de la Clairvoyance, Bonne Entreprise, Prudente Victoire, Image, Créatrice d'Évidence, Lame Miroitante, Fléau de Dieu. (p. 50)

On voit dans cet extrait la part d'incertitude qui entoure la constitution du nom de l'héroïne : on ne peut savoir à coup sûr comment la Pucelle se nommait et on apprend qu'à peu de choses près, elle aurait pu rester sans nom ou être prénommée par sa profession, son gentilé. Cependant, à partir du moment où le mythe se fige, il est possible de le dupliquer en d'innombrables déclinaisons, toutes plus grandiloquentes les unes que les autres. De la même manière, Quintane veut montrer que le corps même de Jeanne d'Arc aurait pu l'empêcher de devenir cette hérö̈ne :

Il suffirait d'une modification minime de son anatomie pour qu'elle soit un peu moins, ou plus difficilement, Jeanne Darc : qu'elle porte des mains tournées à l'envers, pour qu'elle soit contrainte d'inventer une autre manière de prier, - qu'elle ait yeux et bouche enfoncés dans le ventre, pour ne pas pouvoir mettre de casque, - que ses jambes soient aussi flexibles que des lanières, pour ne pas pouvoir monter à cheval, — qu'elle possède une lèvre supérieure immense, pour ne plus pouvoir parler dans les 
négociations, - ou que sa bouche, ossifiée ou trop petite, l'empêche de manger, - qu'elle ait les bras attachés derrière les oreilles, pour qu'elle écrive et se batte avec moins d'aisance. (p. 55)

Cependant, l'histoire a beau être fragile, ses conséquences sont véritables et tangibles: "Grâce aux journées précédentes, nous n'avons pas vécu dans un autre pays, pas habité une autre maison, pas connu un père différent dont le nom surprend, pas pris une robe que nous n'aurions jamais portée, pas dit bonjour en breton. » (p. 39) Si on parle le français, si le pays est homogène, c'est bien parce que la guerre a été gagnée, les institutions protégées, etc. En même temps, il en aurait fallu de peu (une malformation physique, par exemple) pour que la France soit une province anglaise, un duché allemand. Quintane se permet de pousser plus loin son enquête en mettant en scène une interview avec la Pucelle à propos des voix qui la poussent à mener sa guerre. Voici un échantillon de questions imaginées par Quintane :

- Quand elle disait «voix » d'ailleurs, c'était une façon de parler. Mais allez expliquer ce que « voix » veut dire quand ça ne veut pas dire voix.

- Pouvez-vous imiter les voix qui vous parviennent?

- La voix est-elle solidaire d'une image, ou peut-elle retentir seule, sans aucun support iconique?

- Y a-t-il une voix, parlant par la bouche de plusieurs saints, un saint ayant une multiplicité de voix, ou chaque saint avec sa voix propre?

- Se mettent-elles en colère?

- Changent-elles (d'intonation, timbre, débit, localisation dans l'espace) selon la nature de ce qu'elles disent?

- Avez-vous une fois eu l'impression qu'on vous parlait sans voix?

- Les saintes parlent-elles toujours de face, ou se placent-elles parfois de profil?

- Non vierge, seriez-vous en mesure de les entendre?

- Avez-vous eu la tentation de perdre votre virginité : jamais? rarement? (p. 62-63) 
Comme on le voit, Quintane n'en reste pas là : si la trajectoire de Jeanne dépend des voix qui lui parviennent à l'oreille, un phénomène mystique mais étrange, curieux, voire fou, la question de la virginité est aussi centrale. Quintane confronte le mythe avec provocation, pour montrer à quel point la morale y occupe une place majeure. Comme lorsqu'elle indique que plus Jeanne gagne de batailles, plus le mythe de sa virginité gagne en puissance, elle insiste sur le fait que le personnage se désigne comme pucelle et qu'il va même jusqu'à inviter les détracteurs à vérifier : "Encore une chance qu'avec toutes ces chevauchées mon pucelage n'ait pas sauté. Mon nom de pucelle n'est pas le fait du hasard, elles peuvent y mettre les mains, il tient à l'intérieur, et par deux fois il tient. » (p. 63) Et pour montrer que l'histoire, même si elle est fragile, est impitoyable, Quintane met en scène, à la fin de son livre, la dégradation d'une Jeanne capturée, sur le point de se faire juger :

Dégradation matérielle et spirituelle: «Même l'incertitude de la guerre et des partis ne t'ont rien appris. Tu parais devant tes juges telle que tu aurais pu paraitre au sortir de Domrémy. Tu parles par fatigue, sans penser ce qui t'arrive. Aussi bien, tu penses qu'il ne t'arrive rien d'autre que ce que tu avais prévu. Tu es seule; toute une armée n'a pu t'ôter cette solitude. Et quand elle se reporte dans le passé, elle s'y voit, bergère, déjà fatiguée. (p. 73)

L'écrivaine montre la fragilité du personnage, le côté tragique de l'histoire, à quoi rien ne résiste, pas même une armée triomphante. La soldate redevient bergère, avant de monter sur le bûcher où Quintane, dans une belle composition, fond l'avancée de l'armée et des flammes :

Le circuit patiemment construit - de Domrémy à Auxerre, de Gien à Bourges, de Sens à Paris, de Reims à Soissons, d'Arras à Rouen - entame sa dernière progression, des pieds aux chevilles, des genoux aux tendres cuisses, du téton à la nuque, du menton vibrant aux narines frémissantes (et c'est de la bouche que sort la colombe). Lunules ou rotules, plus rien n'est discret, c'est d'un 
corps neuf (vain?) et compact qu'elle flambe, joyeux incendie, méchoui mystique. (p. 77)

Jeanne Darc nous laisse finalement devant cette question : pourquoi Quintane, écrivaine post-avant-gardiste que tout éloigne d'une entreprise historiographique sur ce personnage, s'intéresse-t-elle, à la fin du $\mathrm{XX}^{\mathrm{e}}$ siècle, à un personnage issu du Moyen Âge? À cette figure, que fait dire l'écrivaine sur notre époque? Au-delà des couples oppositionnels mentionnés précédemment, il semble que le binôme le plus intéressant soit celui qui oppose la Pucelle à Nathalie Quintane. Car ce livre parle aussi d'une guerre symbolique à l'intérieur du champ. L'usage de la métaphore militaire illustre la question de l'écriture aujourd'hui tout comme la possibilité du politique dans la littérature contemporaine, à cette époque «où personne ne rit mais personne ne prend plus rien au sérieux ». Le travail de simplification du mythe auquel se consacre Quintane, sa capacité de banaliser plutôt que de glorifier, montre que l'histoire est parfois aussi ordinaire qu'une anecdote. En dépouillant Jeanne d'Arc de tous les préjugés qui l'entourent, l'écrivaine rend le mythe défectueux, irrécupérable pour les radicaux de tout acabit. De plus, en s'appropriant cette curieuse figure de femme (habillée en garçon, vierge, à demi folle), elle prend aussi position en tant qu'écrivaine. On devine bien qu'elle exècre les clichés habituellement associés à la littérature « de femmes »: souffrance, intimité, confession, voilà qui ne veut pas dire pas grand-chose pour Quintane, qui répond violemment à ces poncifs. Combative sans pour autant jouer à la Jeanne d'Arc des lettres modernes, elle s'élève contre la « ringardisation » de l'écrivain femme en choisissant précisément de travailler le ringard, de s'y frotter, mais pour en faire autre chose. En s'attaquant à la Pucelle, Quintane prend en charge l'histoire et la repasse, pas comme les grands-mères le faisaient avec les chemises, mais plutôt avec toute la bellicosité et l'intelligence auxquelles on 
s'attend de la part d'une écrivaine qui travaille à modifier le devenir d'un champ.

\section{Repasser l'Histoire pour contrer l'oubli}

Ayant d'abord trouvé l'histoire sur son chemin par accident, en étudiant la chaussure, Quintane, avec les années, en fait son objet de prédilection. Les deux livres qui précèdent Tomates (paru en 2010) attaquent de front la mémoire d'événements historiques aux allures de traumatismes collectifs pour la France. Cette évolution vers des sujets plus «graves », sans toutefois que l'écriture ne se rigidifie, est attribuable à de nombreux facteurs : démarche d'ouverture sur le monde sans les bons sentiments que ce genre de processus suppose habituellement, prise en charge de plus en plus grande du rôle « civique » de l'écrivain, nécessité toujours renouvelée de travailler sur de mauvais sujets, ceux qui se trouvent dans les coins sombres de l'histoire nationale, où meurtres et trahisons se mêlent. Cavale et Grand ensemble sont d'excellents exemples du choc que Quintane contribue à créer entre la littérature et le monde, choc redonnant à l'écriture, à défaut d'une efficace réelle, une puissance qui rend aux écrivains la possibilité de damer le pion aux forces d'ordonnancement des corps, de la mémoire et du langage.

Cavale est un roman complexe qui raconte la fuite de l'oncle de la narratrice à travers la Picardie et la Californie en vélo. Il tente de s'éloigner le plus possible de la scène d'un crime xénophobe qu'il a commis : il a tué un Russe avec une boule de bowling. Le roman reproduit la confusion des pensées, des discours, la cavale du protagoniste étant sans cesse interrompue par le soliloque de personnages tous plus délirants les uns que les autres. L'aspect le plus intéressant du roman concerne effectivement le désordre, mais pas 
celui qui superpose les discours, plutôt celui qui «mixe» des scènes historiques, ici encore repassées, mais sur le mode de la reconstitution, comme l'avoue Quintane elle-même : «Vous comprenez, il faut insister sur le fait qu'il s'agit de reconstitutions. » (2006, p. 167) En effet, parce que « le tribunal de l'Histoire n'a pas besoin d'historiens» (2006, p. 159) mais « du souvenir live» (p. 159), Quintane reprend des scènes historiques qui n'ont rien de très glorieux pour la France. Ces souvenirs en direct comprennent une grande part d'imprécision, assumée par l'écrivaine, qui dit préférer l'incohérence de la mémoire à l'ordonnancement violent de l'histoire, qui dénature, assujettit. Deux exemples méritent qu'on leur accorde un peu plus d'attention.

Dans un des 21 débuts de roman proposés dans la première partie de Cavale, Quintane donne à lire une scène triple où les personnages et les temporalités varient dans une fluidité qui ne fait qu'accentuer la confusion. En voici un extrait, assez long, pour qu'apparaisse la force de ce que Quintane y tente :

Bonnot est assis à l'arrière de la traction, le colt pointé sur les flics, son acolyte à ses côtés, il balaye l'immeuble de la planque d'une rafale laissant dans le plâtre des impacts qui en préfigurent d'autres laissés, eux, par la Gestapo trente ans plus tard, des types hurlent dans les caves, ça se fait avec l'aval de Laval, un portrait du maréchal - une vraie croûte, c'est lamentable — est pendu audessus du bureau du chef qui a une sale tête, Bonnot en abat deux dans la nuque (est-ce qu'ils s'en privent, eux?) et un d'une balle qui lui forge un trou parfait en plein front sans débordements façon tulipe, le flic se prend bizarrement le ventre à deux mains, se tortille en tous sens, pose un genou à terre comme au ralenti, s'affaisse sur un coude, puis tache la rue d'un ruisseau de sang, vive l'anarchie! crie son collègue par la portière avant de se recevoir une claque magistrale par le patron - eh, connaud, ils étaient pas sûrs que c'était nous, maintenant, ils en sont sûrs pendant ce temps-là, le chef est au téléphone, il attend les instructions, il demande ce qu'on fait des gars là en bas parce qu'avec tout ce grabuge la presse va rappliquer, la presse rapplique 
quand on le lui dit, pour le moment elle est occupée par le retour de de Gaulle, tout le monde est soulagé, il va remettre de l'ordre, et c'est pas un quarteron de généraux à la retraite qui vont renverser la République, quant aux gars en bas, c'est que des communistes, on leur fait un peu peur et on les relâche, sauf Robert dit Bob, qui est vraisemblablement un porteur de valise de première catégorie, oui, c'est ça! Faites-leur leur fête à ces sales cocos, et vive l'anarchie! mais tu vas te taire, connaud! tu vas la fermer ta grande gueule! où que t'es allé le pêcher, çui-là! comment veux-tu qu'on monte au braco avec un abruti pareil! eh c'est pas moi, c'est toi qui m'as dit de prendre Robert dit Bob, qu'il avait fait ses preuves, qu'y en avait pas deux comme lui pour deviner un coffre! ça fait rien, j'aime pas les convertis! tu le renvoies chez sa mère et c'est marre, fonce! d'accord, on extrait le gars et on le fait cracher, tu parles qu'il était pas à Mostaganem il y a deux ans, vous me donnez l'aval ou vous me le donnez pas? allez-y, tous les moyens sont bons pour défendre la République, et c'est certainement pas un quarteron de généraux en retraite - vous l'avez déjà dit, mon général - OUI ET ALORS si je veux le redire je le redirai, et c'est pas vous qui allez me faire taire, personne me fera taire [...]. (2006, p. 16-18)

Le début de la scène nous plonge dans les années 1910. Nous sommes aux côtés de la bande à Bonnot, groupe anarchiste français qui perpétue plusieurs vols de banques au début $\mathrm{du} X \mathrm{XX}^{\mathrm{e}}$ siècle. Anarchistes méprisant l'ordre établi, ils sont des avant-gardistes du crime (utilisation de la voiture, assassinats des policiers). Quintane met en scène un Jules Bonnot tirant une rafale sur un mur de plâtre, et le récit bascule immédiatement au début des années 1940, sous le régime de Vichy : ce même immeuble est occupé par la Gestapo, qui y torture des résistants, avec «l'aval de Laval». Puis, Quintane propose un plan (au sens cinématographique) sur le supérieur de ce dernier : on voit un portrait chromo du maréchal Pétain. On retourne ensuite au début du siècle, où Bonnot tire sur trois policiers, ce qui fait crier de joie un complice, au grand dam du chef qui ne veut pas se faire repérer. On bascule de nouveau durant la Seconde 
Guerre mondiale : un fonctionnaire se demande quoi faire avec les prisonniers et on évoque le retour de de Gaulle, ce qui ne fonctionne pas historiquement (le général débarque à Courseulles-surMer seulement en 1944). Cela semble incohérent, confus, à moins que Quintane n'évoque son deuxième retour, celui de 1958, en pleine guerre d'Algérie, alors qu'il apparaît comme le seul pouvant sortir le pays de la crise, le général étant appuyé dans une confusion généralisée et bien plus grave que celle que Quintane met en scène (on le voit à la fois comme sauveur de l'Algérie française et comme celui qui va décoloniser ce pays). L'entremêlement temporel est égal à celui des ennemis de la République : les généraux tentant un putsch pour garder l'Algérie française, les résistants prisonniers de la Gestapo, les anarchistes de Bonnot, puis enfin un prisonnier provenant de Mostaganem, là même où de Gaulle prononcera, pour la dernière fois, en juin 1958, les mots suivants : «Vive l'Algérie française! » Parce que « tous les moyens sont bons pour défendre la République », il importe peu que ses opposants soient du bon ou du mauvais côté. Après tout :

Vous n'avez plus qu'à retourner chez votre mère, Robert, on est en temps de guerre, au cas où vous ne le sauriez pas! - oh, des événements, tout au plus, une petite pacifi - C'EST MOI QUI DÉCIDE SI C'EST LA GUERRE OU PAS, et c'est certainement pas un quarteron, je vous jure, patron, je vous jure, j'ai vu le flic tomber, là, sur la chaussée, et c'était mon premier, patron, mon premier, c'est vrai que je sais ouvrir un coffre, c'est mon papa qui me l'a appris, mais un flic, non, je savais pas, alors j'ai pas pu m'empêcher, j'étais trop content, quoi, oui, ben la réclame, c'est moi qui m'en charge, Bonnot Jules [...]. (p. 18-19)

On peut bien dire «à la guerre comme à la guerre », encore faut-il pouvoir employer le terme : il faut rappeler que jusqu'en 1999, on a utilisé « événements d'Algérie » pour parler de ce conflit qui a duré huit ans et fauché la vie de plusieurs centaines de milliers de personnes. Par sa reconstitution, Quintane nous fait revivre cette indé- 
termination propre à tout événement en train d'advenir : en nous projetant dans un lieu qui précède l'œuvre de pacification de la mémoire qu'est souvent l'histoire, elle met celle-ci en scène alors que les rôles ne sont pas encore distribués, figés éternellement :

putain, chef, l'enfoiré, il a avalé sa langue et il est en train de tourner l'œil, qu'est-ce que je fais? Arrose-le Robert, arrose, et ramène-le dans sa cellule, avec tes conneries on peut plus rien en tirer, ah j'en étais sûr, il fait partie du groupe de Bob, ils ont déjà liquidé trois Chleuhs et fait sauter deux trains, ces terroristes, encore des Juifs communistes, j'en informe le maréchal [...]. (p. 19)

Dans cette scène de torture, il y a confusion entre des sympathisants de la cause algérienne et des résistants (coupables d'avoir " liquidé trois Chleuhs », c'est-à-dire des Allemands, peut-être des soldats du III $^{\mathrm{e}}$ Reich, d'avoir saboté des trains, etc.). Quintane oppose donc au moralisme du devoir de mémoire, au révisionnisme léger qui fait qu'aujourd'hui on peut avoir l'impression qu'une grande majorité de Français furent résistants, des événements qu'on préfèrerait nier. En effet, l'histoire de France a son côté sombre, et cela ne date pas d'hier, comme se le remémore un des anarchistes :

je pense à la Commune, voilà comme on les venge, nous, les Communards, et voilà comment qu'on va la leur ramener, la Commune, tous les morts de 70 debout sous leur sale nez morveux, tout ensanglantés mais bien vivants doigt tendu pour désigner leurs bourreaux et les fils de leurs bourreaux, et les petitsfils, on les oubliera pas ceux qui nous ont collés dos au mur, ou parqués dans des trains, ceux qui nous ont couchés dans des caves [...]. (p. 21-22)

Répression de la Commune, collaboration avec les nazis (donc répression de la résistance), torture de sympathisants des mouvements de décolonisation, Quintane renvoie au visage d'une France un peu trop oublieuse le fait que ses dirigeants ont aussi été du côté des bourreaux. L'écrivaine revient à 1870, aux massacres de la Com- 
mune comme à l'humiliation française devant Bismarck, dans un second exercice de reconstitution alors qu'elle revisite la scène de la signature de l'armistice de 1918 :

Mettons-leur sur la gueule jusqu'à la dernière minute, à ces Prussiens, on va leur faire payer 70, garanti sur facture, ou alors tactique : on leur met sur la gueule jusqu'à la dernière minute, comme ça ils s'affolent pour signer à nos conditions et c'est tout bénef. (p. 146)

C'est un autre maréchal, Foch cette fois, qui parle. La guerre de 1914-1918 trouve ses racines dans celle de 1870, Quintane ne se fatigue pas de le faire répéter à ses personnages, comme si la fatalité historique demandait qu'on cogne inlassablement sur le même clou :

Pourrions-nous, Monsieur le Maréchal, suspendre les hostilités, afin de préparer sereinement la paix? Mais bien entendu! Quelle question! Je vous prie (il les précède), on va nous servir une boisson chaude. Ça ne sera pas de refus, il fait plutôt frisquet par chez vous; pire que Strasbourg en 70, ah ah! (p. 149)

Cette allusion est évidemment là pour rappeler que dans ce wagon se prépare déjà une prochaine guerre, la pire de toutes, malgré cette déclaration de l'interlocuteur allemand: «Heureusement pour l'Europe et pour la paix, Maréchal, nos valeureux soldats ne se dévoueront désormais plus qu'à des tâches humanitaires. Il nous faut penser à panser nos plaies, avant tout! » (p. 150)

Ayant souvent pointé la contingence de l'histoire, Quintane montre dans Cavale la tragédie de son inconscience, de son incohérence, de son inconsistance. Il n'y a pas de vérité qui ne repose sur un ordre violent. L'histoire des vainqueurs, quels qu'ils soient, est toujours une entreprise de mise à mort de la mémoire des vaincus. À un journaliste qui lui demande, à propos de ce roman, si « la fuite » est une «solution à la déprime », Quintane répond : «Non, 
si fuite il y a, je l'ai dit, c'est plutôt celle des idées. Mais, certes, si on veut fuir au sens concret, c'est peut-être aussi parce que nos idées fuient. C'est un compte rendu de l'ambiance actuelle, ce dont tout le monde parle sans parvenir à le définir vraiment. » (Loret, 2006, p. 3) Le journaliste désire alors qu'elle précise, lui demandant si elle fait référence à la «situation politique actuelle ». Réponse de l'écrivaine :

cela vient des discussions bizarres que je peux avoir avec des amis à propos de ça, on a la quarantaine, on est éduqués et pourtant il y a des moments où c'est pire que l'affaire Villepin-Sarkozy, c'est le marécage mental complet. Cette difficulté à penser l'avant, l'après, le pendant. Mais je crois que c'est une époque bénie pour moi, concernant la fuite des idées. Je retrouve en vrai cette «brouillasse » qui me travaille depuis toujours. Rien n'est sûr. (p. 3)

Époque qui convient aussi à Quintane parce que son écriture, la manière dont elle se déploie, avec son usage de l'incongruité, sa prédilection pour la surprise et l'idiotie, résiste presque intransitivement aux discours simplistes et consensuels si communs aujourd'hui. «Rien n'est sûr », voilà une formule simple pour évoquer «l'irréductibilité de la contingence » (2005, p. 92) dont parlent Deleuze et Guattari. Un appel au scepticisme, à la critique, mais pas au relativisme. Rien n'est sûr, donc, même si l'histoire œuvre à imposer des vérités aseptisées qui ne laissent aucune place à la contestation. Il s'agit d'ordonnancer la mémoire en vue de bâtir des discours de légitimation pour éviter que l'ordre en place ne soit ébranlé. La littérature, on l'a dit souvent, résiste en s'opposant à cet état de fait.

Quintane s'engage en traitant de sujets nuisibles, nuisibles pour son pays comme pour sa personne. Grand ensemble est la prodigieuse démonstration de ce phénomène : l'histoire de France fusionne avec le corps de l'écrivaine. Ce livre, qui met en scène les aberrations 
entourant un événement culturel ayant eu lieu en 2003 et appelé «L'Année de l'Algérie », attaque la rectitude politique et les impératifs du devoir de mémoire qui apparaissent bien souvent comme des exercices bâclés : «Un fantôme nous hante, insatisfait de sa commémoration (L’Année de l'Algérie, 2003) qui le célébra pour mieux l'effacer encore. Ce livre donne corps à ce spectre. » (Grand ensemble, quatrième de couverture) La formule, qui n'est pas sans faire penser à l'incipit du Manifeste du Parti communiste, ne laisse aucun doute sur l'intention de l'écrivaine d'en découdre avec cette entreprise de commémoration où le passé revient dans sa forme la plus édulcorée. En effet, L'Année de l'Algérie ne se préoccupe pas des détails : par exemple, on sert des cornes de gazelle, dessert marocain, même si la célébration concerne le voisin algérien. Les bons sentiments pullulent. Grand ensemble, dont le sous-titre - «concernant une ancienne colonie » — est on ne peut plus éloquent, rassemble des éléments disparates autour de cet événement. Quintane admet y travailler plus que jamais le frustre, ce livre étant une synthèse de son travail sur les dispositifs formels et de thématiques historiques qui l'interpellent. La question de la mémoire y est posée, tout comme celle d'une idéologie du culturel qui s'avère trop souvent la courroie de transmission d'un conformisme stérile, de vœux pieux.

Le «je » de Grand ensemble est plus près que jamais de la personne de Quintane. Il s'agit donc, avant Tomates, de son livre le plus «politique» et le plus «personnel». Bien davantage que Jeanne Darc, qui portait sur la distanciation, Grand ensemble traite l'Histoire (avec un grand $\mathrm{H}$ ) comme si elle était aussi celle (avec un petit h) de Quintane, les deux corps se liant métaphoriquement par l'écriture, forme de somatisation qui exprime cette violence qui cherche par ailleurs à oblitérer ce qu'il y a de mauvais dans les événements relatés. Voilà pourquoi le livre s'ouvre sur cette anecdote 
apparemment inoffensive : «À la suite d'un herpès occurré à la lèvre supérieure en février 2003 et particulièrement défigurant, je décidai d'écrire un texte intitulé L'Année de l'Algérie. » (2008, p.9) Cette inflammation soudaine coïncide avec le début de la célébration officielle. Des microrécits, nombreux, concernent le père de l'écrivaine. Celle-ci relate une séance de diapositives où, trop myope, elle observe les photos de paysages prises par le père ou encore des objets ramenés d'Algérie : « une broche en émail bleu en forme de croix du Sud avec en son centre en contre-plongée un Touareg, et une enveloppe de la Poste aux Armées car il y travaillait étant trop myope pour la guerre » $(2008$, p. 18) Le père et la fille Quintane sont peut-être tous les deux trop myopes, mais cela n'empêche pas l'écrivaine de poser un regard sur les horreurs de son histoire nationale, d' " évoquer une torture sans passer par l'oxymoron » (p. 11). En effet, Quintane affronte l'horreur sans détour, ne la dilue dans aucune figure de style, que ce soit l'oxymore, l'hyperbole, la litote ou l'euphémisme, qui fait parler de cette guerre comme d' « événements »: « couilles coupées portées encore chaudes à la bouche. Électrification non des campagnes. Cuts de langues. Enculades non toujours de petits garçons. » (p. 12) La violence, la torture, voilà ce que Quintane repasse (comme on le dit d'un mauvais film, à la télévision), sans complaisance, donnant droit de cité à ce qui se cache derrière l'entreprise commémorative défectueuse mise en place par le gouvernement. Ce repassage n'est pas ici une mise à plat mais plutôt une mise en relief de ce qui justement est trop aplati. Quintane en parle en ces termes :

La rétrospection est un travail. La rétrospection est notre travail car le présent ne suffit pas. Comment agir tout court par le biais de la rétrospection? Comment agir par le biais de la rétrospection sans être traité de marxiste intellectuel pédophile? Il s'agit, au moment d'agir, de travailler à mieux prêter le flanc aux accusations de marxiste intellectuel pédophile. (p. 106-107) 
Regarder le passé n'est pas chose simple, mais c'est à la littérature de faire ce «travail », malgré les accusations farfelues auxquelles Quintane invite à tendre la joue. La démagogie n'interfère pas avec l'écriture mais la nourrit. L'oubli est le chemin facile, parce que, dans ce cas précis, se souvenir consiste à travailler dans la tristesse et dans la mort : "Que les événements produisent des larmes, c'est ce que nous prenons garde d'oublier. Ainsi sommes-nous perpétuellement surpris, enchantés et curieux. Enchantés, curieux et désolés, notre cœur se ravive à l'arrivée des larmes : nous vivons. » (p. 109) Travailler quelque chose de mauvais est exigeant, amène à pleurer, mais témoigne au moins de la vie comme résistance, résilience même à la dénégation d'un passé traumatique. Et c'est précisément parce que sa pensée est si vive que Quintane peut parvenir à ce qui paraît impossible : évoquer de terribles drames tout en nous obligeant à esquisser un grand sourire :

Une femme terroriste: double difficulté. Une femme terroriste infirmière : triple difficulté. Une femme donne la vie, une femme terroriste donne la mort : contradiction. [...] Mais en même temps, si elle se tue au moment même où elle donne la mort, c'est peutêtre qu'elle ne supporte pas de la donner, qu'elle ne veut être dans la possibilité de pouvoir constater la mort des autres et donc qu'elle ne veut plus être vivante quand ils seront décédés : c'est que c'est une femme, et de plus une infirmière. Une infirmière morte n'a plus la capacité de soigner les gens qu'elle a tués - et heureusement, car son métier lui commanderait, une fois qu'elle s'est jetée contre l'autobus munie d'une barrière de dynamite, de se relever d'entre les morts et d'y dénicher un vivant au moins, de vérifier le pouls de ce vivant tout en pestant : même pas mort, qu'est-ce que c'est que ce travail. (p. 125-126)

Cette logique un peu tordue lui donne l'occasion de montrer l'aporie que constituent les attaques kamikazes aussi bien que n'importe quel documentaire ou étude : en mettant en scène cette situation improbable où l'infirmière terroriste se dédouble et tente 
de venir en aide à ses propres victimes, et en critiquant l'amateurisme du travail de meurtrière de cette dernière, Quintane confirme que seule la littérature peut engendrer une telle inventivité, qui donne au tragique un autre éclairage qui résiste au black-out de la dénégation. Il faut penser autrement, penser à partir de l'extérieur mais penser, critiquer, faire voir ce que d'autres veulent voir s'effacer. Solliciter la référence la plus saugrenue pour témoigner d'une situation bien plus déraisonnable encore :

Chan, acculé par quelques bagarreurs classiques dans un appartement modèle, utilisait les meubles comme autant de camarades, basculant assis dans un fauteuil pour mieux pincer un nez à droite, une joue à gauche, en balançant. Chan marchait au mur, et le mur n'était pas son ennemi. Rien n'était à l'opposé de Chan, tout était avec lui, ou devait l'être, par sa grâce. Tremblement de terre? Tremblement de terre. Concert de Jane Birkin? Concert de Jane Birkin. Menace de crise? Menace de crise. Queue devant les postes à essence? Noces devant les postes. Trop de pots de yaourt? emploi des pots. Poubelle? invention d'une synthèse aromatique. Chan gagnait. Une terrible nostalgie m'advint : Chan aurait pu gagner. Nous aurions pu tuer en dansant - au lieu de porter des bardas de soixante-quinze kilos et de faire des pompes en disant: "C'est la vie de château, merci mon adjudant. » Enfoncement des portes à coup de godasse. Cramage de mechtas. Enculages non toujours de petits garçons. Impensable vulgarité des chars, des bazookas, des lance-flammes, des drones. Minoration par le design - élégance d'un bazooka, angle de pénétration du char dans l'air, géométrie magnifique des drones. (p. 138)

Malheureusement, l'invention se déploie au mauvais endroit. On aurait pu faire la guerre avec style, avec grâce. Mais tout ce qui s'est passé en Algérie s'est fait vulgairement. Il ne s'agissait pas d'un film de Jackie Chan, certes, mais on pourrait se rappeler ces événements avec justesse et mesure plutôt qu'avec l'excès inverse, qui consiste à mettre en scène à la bibliothèque municipale et au cinéma du centre culturel des événements lénifiés : «Lire en fête, 
l'Algérie, 9 au 22 octobre, rencontre, littéraire, conférence, exposition, ateliers, gastronomie, contes, cinéma. » (p. 64) Évidemment, le problème n'est pas de célébrer un pays ami, mais de faire abstraction de la litigieuse histoire qui le lie à la France. C'est ce qui est mis en lumière par cette double remarque de Quintane revenant sur une anicroche survenue lors d'une " célébration »: "Demande de précision concernant la très brève évocation de la nuit d'octobre » (p. 67); «Demande de précision sur la raison de la très grande brièveté de l'évocation de la nuit d'octobre. Que non, c'est une fiction, et que le film ne porte essentiellement pas sur la nuit d'octobre. » (p. 67) Il est facile de parler de gastronomie et de coutumes régionales, mais un massacre ${ }^{2}$ dans les rues de Paris, c'est plus compliqué. Compliqué parce que la situation n'est toujours pas rentrée dans l'ordre, comme en témoigne cet exemple aux allures anodines mais qui rappelle la précarité économique des Français d'origine algérienne, sans parler de la situation explosive dans certains quartiers chauds :

Je veux bien débourser cinquante balles pour une heure de ménage, en plus elle ferait mieux le ménage que moi, cette présentatrice de la télévision algérienne a certainement une femme de ménage, si la plupart des femmes de ménage en France sont de nationalité algérienne, quelle est la nationalité de la plupart des femmes de ménage en Algérie? mettons que les femmes de ménage viennent toujours plus du Sud, en Algérie elles seront de nationalité béninoise par exemple, mais au Bénin? au Bénin elles seront donc de nationalité zaïroise, et au Zaïre? elles seront Angolaises, par exemple si elles ne sont pas toutes décédées dans la guerre ou dans le sida, et en Angola? elles viendront, noires, d'Afrique du Sud sans aucun doute, et en Afrique du Sud? eh bien elles viendront de l'Antarctique. (p. 24-25)

${ }^{2}$ Au sujet du Massacre du 17 octobre 1961 et de Maurice Papon, voir le roman de Didier Daeninckx, Meurtres pour mémoire, Paris, Gallimard, 1984. 
Quintane évoque la dénégation violente du discours de l'époque, à mettre sur le dos du colonialisme : «D'ailleurs, il y avait des éléments curieux concernant ce pays grand (mais petit, dans une Afrique gigantesque) : l'Algérie avait été un département français au même titre que la Saône-et-Loire! Le Maroc et la Tunisie non, mais l'Algérie oui, et c'était tout naturel. » (p. 131) Malgré la double aberration historique (faire de ce pays, mais pas des autres, un département; contenir un territoire si immense dans une structure administrative disproportionnée), c'est tout logiquement que cet état de fait se produit :

Comme si la guerre avait consisté huit ans à battre l'air autour de vous, rien. Le département signifie qu'on est à l'intérieur, division (d'un corps grand) inexpulsable. L'intérieur ne peut pas être à l'extérieur, disent les colons, et logiquement ils n'ont pas tort. Le colon dit : l'Algérie est un pays logique. (p. 132)

Évidemment, la force du travail de rétrospection entrepris par Quintane réside aussi dans sa capacité de dresser une continuité entre un passé douteux et un présent qui l'est tout autant, malgré sa différence. Premier symptôme de ce passé qui «passe mal »: la régression. Quintane écrit: «Depuis l'indépendance, je suis atteinte d'une méningite généralisée. De lui consacrer une année civile, de chanter pour elle en tapant des mains. » (p. 132-133) Elle parle ici pour son pays, se dit atteinte d'une «méningite » qui l'amène non pas à évoquer les massacres mais à chanter tout en tapant des mains. L'idiotie, certes, mais au sens presque médical d'arriération quant au fait d'assumer ce qui précède, justement. Ainsi, les appels au devoir de mémoire semblent naïfs dans un contexte où la prise en charge de l'histoire rappelle à Quintane un exercice stérile pour se donner bonne conscience :

Rien, sinon le compost clean qui ne fait rien pousser et sur lequel on trône. Ce compost de vieilles formules toutes devisant de dévoilement tandis que le premier désir d'un nouveau maire ou du 
poète nouveau est d'abattre, de combler, de couvrir et d'édifier. Contentez-vous de l'édification, dit-on à tous les céciteux, à tous, puis on les somme. En parallèle aux dormitions se déplient une à une les horreurs, comme en un monde séparé, comme en un jadis. (p. 116)

Salmigondis de phrases creuses, qui oblitèrent, en même temps qu'elles sont énoncées (par le mauvais politicien, le tout aussi mauvais poète), les squelettes qui débordent du placard. Une cécité de l'ordre de l'injonction, pour tenir l'abject, le douteux, loin de soi, dehors, comme si une telle volonté d'effacer n'en disait pas autant que ce qu'on cherche à taire. Les morts de l'Algérie appartiennent peut-être à un autre temps, mais ils parlent encore du nôtre (Grand ensemble se clôt sur des épitaphes de martyrs récents), même si le pouvoir et ses malversations semblent aujourd'hui moins repérables, plus furtifs, un peu comme le spectre politique :

Il n'y a plus ni gauche ni droite. Il n'y a plus démocratie parlementaire ou démocratie non parlementaire, ou monarchie constitutionnelle, ou monarchie parlementaire, ou despotisme éclairé, ou dictateur éclairé, ou dictateur, ou démocratie socialiste, ou dictature communiste, ou dictature communiste parlementaire, ou monarchie socialiste éclairée, ou fédéralisme constitutionnel démocratique. Le monde est divisé entre éradicateurs et dialoguistes. (p. 104)

D'un côté, une violence qui n'appelle rien d'autre qu'une éradication (toute résistance étant semblable à une maladie), de l'autre, des invitations au dialogue qui ne font que jouer le jeu du statu quo. Pourtant, le passé guette un présent dont la volonté de clarté n'empêche en rien une dérive dans la confusion :

Le raidissement des années 00 énonce la crainte du confus, quand en fouillant les dossiers d'une grand-mère, on découvre bien ordonnés des discours d'Henriot, le maréchal serrant les mains, une photographie noir et blanc du jeune de Gaulle, Mitterrand assurant l'Algérie française, chaque pensée française dans une tête française donnant du mou. La France a nazillonné. Pas plus 
possible de passer ça qu'un protestant les couilles, nez, oreilles, enfilés en guirlande aux chapeaux catholiques. Explication par papa : en face ils faisaient pire, ou : ils faisaient pareil, quand tu retrouves un copain les couilles dans la bouche qu'est-ce que tu fais. Et qu'est-ce que tu fais quand tu entends ça. (p. 145-146)

«Qu'est-ce que tu fais quand tu entends ça? », nouvelle déclinaison de la fameuse remarque d'Adorno («écrire un poème après Auschwitz est barbare »), adaptée au début du XXI ${ }^{\mathrm{e}}$ siècle. C'est «la question qui tue », celle qui embarrasse, que n'importe quel écrivain qui veut travailler le politique aujourd'hui doit affronter. Le constat de Quintane est indéniable : la France a collaboré, puis a participé à une guerre coloniale terrible, à des actions, des exactions dont la barbarie n'a rien à envier aux guerres ayant opposé catholiques et protestants. Quoi faire, demande Quintane? Ou plutôt, comment faire avec, comment produire de la littérature à partir de ça? Il faut repasser. Jeanne Darc, Cavale et peut-être de façon plus frontale (et plus efficace?) Grand ensemble sont des réponses à la grave question posée par Nathalie Quintane. Dans un pays qui a mis autant de décennies à faire preuve d'un tant soit peu de regrets, dans un contexte politique où Nicolas Sarkozy, le soir même de sa prise du pouvoir, a affirmé devant des dizaines de millions de Français que la repentance est une forme de «haine de soi », force est d'admettre que le combat est loin d'être gagné, qu'il faut user (pour paraphraser l'expression que François Mitterrand, ministre de l'Intérieur en 1954, consacrera) de «tous les moyens » donnés à la littérature pour se rappeler à quel point il est facile d'oublier. 


\section{Bibliographie}

DeleuZE, Gilles et Félix GUATTARI. (2005), Qu'est-ce que la philosophie?, Paris, Minuit

DUCHET, Claude. (1979), Sociocritique, Paris, Nathan

LORET, Éric. (2006). « De la suite dans les idées fixes », Libération, cahier Livres, 11 mai, p. 3-5

Quintane, Nathalie. (1997), Chaussure, Paris, P.O.L

-. (1998), Jeanne Darc, Paris, P.O.L

-. (2001), Saint-Tropez - Une Américaine, Paris, P.O.L

-. (2004), Antonia Bellivetti, Paris, P.O.L

-. (2006), Cavale, Paris, P.O.L

-. (2008), Grand ensemble, Paris, P.O.L

\section{Résumé}

Plutôt qu'un programme, la littéralité chez Nathalie Quintane est un procédé et une posture à partir desquels elle retravaille la matière historique. Plusieurs de ses œuvres "repassent» l'Histoire officielle de la France, soit en aplatissant des personnages et événements mythifiés à des fins idéologiques dans de grandiloquents récits, soit en mettant en relief des "mauvais sujets » ou des épisodes peu glorieux omis par cécité volontaire. De la même manière que la figure de "Kroutchev » est éclipsée dans Chaussure par le soulier avec lequel il tape sur la table de l'Assemblée générale de l'ONU, Jeanne Darc déboulonne la machine à fiction de la pucelle guerrière par la mise en exergue des 
contingences bêtes qui la font passer de bergère consciencieuse à soldate. Alors que Cavale montre l'inconsistance et la confusion de la mémoire dans la construction de la réalité historique, Grand ensemble en découd avec la commémoration édulcorée des " événements » d'Algérie en disant crûment l'horreur cachée par les récits officiels. L'œuvre de Nathalie Quintane est exemplaire du rôle politique de la littérature dans la résistance à l'ordonnancement de la mémoire.

\begin{abstract}
The political dimension of Nathalie Quintane's work goes beyond her positioning in the restricted literary field. Rather than a program, her literal poetry is a device and posture allowing her to question and reshape the historical matter. Her writing revisits France's official History, either by flattening characters and events mythicized into bombastic narratives to serve ideological means, either by showing up "bad subjects" or unglorious episodes voluntarily hidden or forgotten. As does Chaussure with the obliteration of Khrushchev's character behind his slamming of a shoe on the UN General Assembly table, Jeanne Darc debunks the virgin warrior portrayal's fictionalisation by showing the silly contingencies in the making a soldier out of a conscientious shepherd. Whereas Cavale shows the inconsistency and confusion of the memory in the construction of the historical reality, Grand ensemble fights the toned-down commemorations of the Algeria "events" by crudely telling the horrors behind the official stories. Nathalie Quintane's work is exemplary of the political role played by literature in resisting the organization of memory.
\end{abstract}

